\title{
Julie D. Saba: pediatric oncology is an amazing field that requires both vigilance and medical acumen
}

Submitted Jul 03, 2018. Accepted for publication Jul 04, 2018.

doi: $10.21037 /$ tcr.2018.07.03

View this article at: http://dx.doi.org/10.21037/tcr.2018.07.03

\section{Editor's note}

Every day of pediatric oncologists is filled with challenges and a quest to win the day. With a persistently escalating number of pediatric cancer incidence across the world over recent decades, it is believed by many that it is not merely due to the improvements in diagnosis, but also a genuine increase in underlying risk. Statistically, it is estimated that over 175,000 children develop cancer annually in the world, with a mortality rate of approximately 96,000 per year, according to a study of Children With Cancer UK (1). In spite of improved prognosis in pediatric cancers thanks to novel drug therapies, drug delivery systems, and protocoldriven treatments that help standardize therapy, pediatric oncology remains a challenging yet stimulating field with plenty of research opportunities and discoveries every day.

As the John and Edna Beck Chair of Pediatric Cancer Research at USCF Benioff Children's Hospital Oakland and Adjunct Professor of Pediatric Hematology/Oncology at the University of California San Francisco (UCSF), Prof. Julie D. Saba (Figure 1) has been leading a series of pediatric cancer research studies to examine the genetic forces driving pediatric malignancies, colon carcinogenesis and other pediatric deficiencies, as well as to develop new therapeutic strategies to treat these pediatric diseases. In this interview, Prof. Saba will share with us some interesting facts, findings and stories with regard to her pediatric cancer research.

\section{Expert introduction}

Julie D. Saba, MD, PhD, currently serves as the John and Edna Beck Chair of Pediatric Cancer Research and Director of the Swim Across America Cancer Lab at UCSF Benioff Children's Hospital Oakland. She is also an Adjunct Professor of Pediatric Hematology/Oncology at the University of California San Francisco, California. She is a pediatric oncologist and senior scientist at the Children's Hospital Oakland Research Institute (CHORI), where she and her team have been studying pediatric malignancies, colon cancer, muscular dystrophy, T cell trafficking, and the

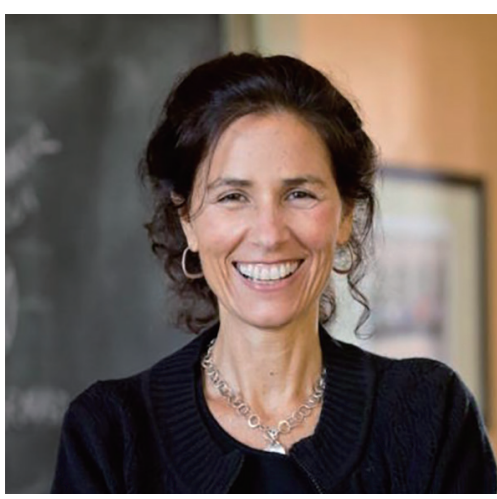

Figure 1 Prof. Julie D. Saba.

newly recognized inborn error of metabolism called SPL insufficiency syndrome (SPLIS).

Prof. Saba has been actively involved in multiple academic societies. She is a member of the Society for Pediatric Research, the Children's Oncology Group and the American Society for Clinical Investigation. She is also a member of the Chemo and Dietary Prevention of Cancer Study Section of the National Institutes of Health. Prof. Saba has received a number of eminent awards and honors such as being the STEM Woman of the Year of CA Assembly District 15 in 2014, and obtaining the "CHORI Lifetime Scientific Achievement Award" in 2016.

\section{Interview}

TCR: The Pediatric Cancer Research team you led at CHORI has recently discovered an S1P-regulated gene that drives the growth and tumorigenicity of highly malignant pediatric tumors. What is your current understanding about this gene?

Prof. Saba: Sphingolipids represent a conserved family of lipids that are present in the plasma membranes of all of our cells. They can be broken down to metabolites that have biological activities, and one of these metabolites is called sphingosine-1-phosphate (S1P). S1P binds to a family of G protein coupled receptors that transmit signals regulating 
cell growth and cell migration. We showed previously that the metabolism of S1P could control cell transformation, the process by which cells begin to acquire a malignant phenotype, growing faster and being able to form tumors in immunodeficient mice. We wanted to learn more about the way that S1P drives proliferation. We conducted a simple study to look for genes that are upregulated or downregulated when the S1P levels of the cell are chronically altered. This study led us to the discovery of an S1P-regulated oncogene that was previously identified in an infant's leukemia cell. Subsequent analysis of a variety of pediatric tumor samples revealed that this gene is highly expressed in neuroblastoma, a deadly childhood cancer. Our current studies are focused on understanding how this oncogene drives carcinogenesis in neuroblastoma. Since this work is not complete, we cannot describe it in detail. However, at least one mechanism involves the oncogene's ability to prevent cell senescence, or aging.

TCR: Are there any current studies aiming to develop new therapeutic strategies targeting S1P and other bioactive sphingolipids in treating pediatric malignancies?

Prof. Saba: S1P is generated by an enzyme called sphingosine kinase, which has two isoforms. There are five known S1P receptors that mediate S1P signals. Both sphingosine kinase and S1P receptors are excellent targets for therapeutic modulation of S1P signaling, and there is considerable effort in the pharmaceutical space to develop specific inhibitors to sphingosine kinase and antagonists of S1P receptors for therapeutic purposes. Since S1P signaling regulates lymphocyte trafficking, these drugs have been effectively used to treat multiple sclerosis and other autoimmune diseases. However, the role of these drugs in the treatment of cancer is not fully realized and will become more evident with time.

TCR: It's been said that genetic mutations are the "match that lights the fire" of cancer, and inflammation is the "fuel that feeds the flame". How does it reflect the situation of colon carcinogenesis?

Prof. Saba: Virchow is the German scientist who is attributed to first recognizing the link between cancer and inflammation. For many years, it was believed that the mutations "light the match", i.e., start the process of cell transformation that leads to malignancy and that inflammation "fuels the flame", i.e., makes established tumors grow faster. However, more recent discoveries show that signals emanating from STAT3, NF- $\kappa \mathrm{B}$ and S1P drive cell transformation and can jump start the carcinogenic process. It is now more challenging to say where cancer actually begins versus what promotes the oncogenic process, since inflammatory pathways seem capable of "lighting the match" themselves.

\section{TCR: Your findings suggest that dietary sphingolipids} may enhance or inbibit colon carcinogenesis depending on whether they can be metabolized to S1P. Would you explain the mechanisms bebind?

Prof. Saba: Dietary sphingolipids come in a variety of forms and are structurally diverse. Sphingolipids derived from animal food products look like those in our bodies and are based on a sphingosine backbone. Sphingosine is taken up by colon epithelial cells and can be turned into $\mathrm{S} 1 \mathrm{P}$, which drives inflammation and carcinogenesis, which is obviously not good for us. Normally, enzymes in the gut mucosa rapidly degrade any S1P that is produced. However, that is now always the case. Sphingolipids derived from soy and some other natural sources are structurally different and suppress cancer signaling pathways that can drive tumorigenesis. We believe that soy sphingolipids have chemopreventive properties.

\section{TCR: Would you introduce us to a most recent NIH research that you are involved in?}

Prof. Saba: We were just awarded an NIH R01 grant to study in greater depth the link between S1P signaling and metabolism and colitis. The connection between inflammation and carcinogenesis is nowhere more evident than in the high incidence of colon cancer in patients with inflammatory bowel disease, a category of inflammatory conditions comprised of ulcerative colitis, Crohn's disease and indeterminate colitis. We are trying to understand how S1P signaling and metabolism contribute to this process. This grant is a standard R01 but was administratively cut by one year and one module. It will last for four years and provides $\$ 225,000$ in direct funds to my laboratory for this scientific study. The funding should begin this summer.

\section{TCR: What do you regard as the most difficult aspects of doing research?}

Prof. Saba: Research requires being willing to be in over your head all the time. You are constantly probing to get at the truth of something, and if the question is worth answering 
it isn't going to be easy. It takes confidence and a willingness to accept that producing one small advance or revealing one new insight may be all that comes from a year's worth of total dedication. One must cultivate patience and seek to understand one's failures, because all the greatest riches can be found in the negative data. That can be brutally hard to do, but it's the only way to get to the eureka moments.

\section{TCR: Why are you particularly interested in pediatric oncology?}

Prof. Saba: Pediatric oncology is one of the most passionate and demanding fields there is. What can be more heart wrenching than a child with cancer? What medical subspecialty requires more vigilance and medical acumen than pediatric oncology, where a two-year old can come in looking like any normal kid but actually being on the verge of a calamity with neutropenia, thrombocytopenia, anemia and fever? And then you start chemo and they run the risk of tumor lysis syndrome. And then there is the emotional intelligence required to guide anxious parents through the treatment process. And yet the patients themselves are usually the least concerned. As soon as they feel better they just want to play. It's an amazing, amazing field.

\section{TCR: Can you share with us the most memorable clinical cases you bave ever encountered?}

Prof. Saba: One remembers the best and the worst cases. I remember being on call one night and taking care of a terminal patient with a brain tumor. I went into the room and checked on him. He was breathing quietly, but something seemed amiss. I looked around the room and did not see anything unusual until my eyes strayed into the corner of the room where the boy's mother was crouched under a chair. I took her hand and coaxed her out. When she stood up she was tall and immaculately dressed but almost catatonic. I brought her to the bedside of her son and gave her a washcloth and encouraged her to sit down by her son and help him. It was the worst, most primal, terrible thing a mother could possibly go through and I will never forget it. On the other hand, I remember my little leukemia patients riding through the hallways on their IV poles like road hogs, laughing behind their paper masks, bald heads shining.

\section{TCR: In your opinion, what are the key skills or qualities a successful pediatric oncologist must possess?}

Prof. Saba: Optimism, precision, ability to continually change-along with a constantly evolving set of treatment protocols, vigilance, an aptitude for sensing the state of mind of a parent, an ease with people living in a state of high anxiety, a disposition for instilling hope, an easy way of explaining, and a robust capacity for active listening.

\section{Acknowledgments}

We would like to express our sincerest gratitude to Prof. Julie D. Saba for sharing her insights and opinions with us. Funding: None.

\section{Footnote}

Provenance and Peer Review: This article was commissioned by the Editorial Office, Translational Cancer Research. The article did not undergo external peer review.

Conflicts of Interest: The author has completed the ICMJE uniform disclosure form (available at http://dx.doi. org/10.21037/tcr.2018.07.03). The author has no conflicts of interest to declare.

Ethical Statement: The author is accountable for all aspects of the work in ensuring that questions related to the accuracy or integrity of any part of the work are appropriately investigated and resolved.

Open Access Statement: This is an Open Access article distributed in accordance with the Creative Commons Attribution-NonCommercial-NoDerivs 4.0 International License (CC BY-NC-ND 4.0), which permits the noncommercial replication and distribution of the article with the strict proviso that no changes or edits are made and the original work is properly cited (including links to both the formal publication through the relevant DOI and the license). See: https://creativecommons.org/licenses/by-nc-nd/4.0/.

\section{References}

1. Children with Cancer UK [Internet]. The United Kingdom: Children with Cancer UK; c2018 [cited 2018 Jul 3]. Available online: http://www.childhoodcancer2017. org.uk/ childhoodcancer.asp

(Science Editor: Brad Li, TCR, tcr@amepc.org)

Cite this article as: Li B. Julie D. Saba: pediatric oncology is an amazing field that requires both vigilance and medical acumen. Transl Cancer Res 2018;7(Suppl 7):S810-S812. doi: $10.21037 /$ tcr.2018.07.03 